\title{
Using level-2 fuzzy sets to combine uncertainty and imprecision in fuzzy regions
}

Verstraete Jörg

\begin{abstract}
In many applications, spatial data need to be considered but are prone to uncertainty or imprecision. A fuzzy region - a fuzzy set over a two dimensional domain - allows the representation of such imperfect spatial data. In the original model, points of the fuzzy region where treated independently, making it impossible to model regions where groups of points should be considered as one basic element or subregion. A first extension overcame this, but required points within a group to have the same membership grade. In this contribution, we will extend this further, allowing a fuzzy region to contain subregions in which not all points have the same membership grades. The concept can be used as an underlying model in spatial applications, e.g. websites showing maps and requiring representation of imprecise features or websites with routing functions needing to handle concepts as walking distance or closeby.
\end{abstract}

\section{Introduction}

The concept of the fuzzy regions originated from a need to represent and reason with imperfect spatial information. The available models did not provide ample capabilities deal with imprecision or uncertainty of the data, particularly when the imprecision or uncertainty concerned the locational data itself: a region without a crisply defined outline, a region with elements that only partly belong to it or a point located at an imprecise location. The concept of fuzzy regions solved this, the fuzzy set over the two dimensional domain can be given a veristic interpretation ([1]), where the membership grades indicate the extent to which points belong to the set

Verstraete Jörg

Systems Research Institute - Polish Academy of Sciences, Ul. Newelska 6, 01-447 Warszawa, Poland / Database, Document and Content Management - Department of Telecommunications and Information Processing, Sint Pietersnieuwstraat 41, 9000 Gent, Belgium

e-mail: jorg.verstraete@ibspan.waw.pl, jorg.verstraete@telin.ugent.be 
thus representing a region; or a possibilistic interpretation ([1]) where the membership grades indicate the possibility this point is a valid candidate, thus indicating a fuzzy point. However, in the model, all elements were considered independently from one another, yet sometimes a user can have additional information. An example would be the representation of a lake with a changing water level. All points at the same altitude along the side of the lake will be either above the water or under the water at the same time; so it makes sense to group these points together if we want to represent the lake as a fuzzy region.

In this contribution, we will go deeper into a mechanism that allows such internal dependencies to be modelled. In section 2 we will give a brief overview of the current model for fuzzy regions 2.1.1, the first extension that makes use of the powerset (in 2.1.2) and its limitations (in 2.2). Section 3 concerns the proposed extension to use the fuzzy powerset in order to enrich the fuzzy regions and the resulting interpretation (in 3.3). The conclusion (section 4) summarizes the finding and mentions future work.

\section{Preliminaries}

In this section, the current definition for fuzzy regions and its extension using the powerset of the two dimensional domain is presented.

\subsection{Fuzzy regions}

Traditionally, a polygon or other closed line is defined to be the boundary of a crisp region; the region is then considered to be inside this boundary ([6]). However, it is also possible to consider the region as the set of points contained in this boundary, and this is the view from which the fuzzy region can be defined.

\subsubsection{Definition}

From this point of view, it is a small step to augment the definition to a fuzzy set ([13], [14]) of points. In [8], the fuzzy region was defined over $\mathbb{R}^{2}$, thus with each element (point) a membership grade was associated.

\section{Definition 1 (Fuzzy region).}

$$
\tilde{R}=\left\{\left(p, \mu_{\tilde{R}}(p)\right) \mid p \in \mathbb{R}^{2}\right\}
$$

A fuzzy region essentially is a fuzzy set defined over a two dimensional domain; the concept is illustrated on figure 1. Consequently, the traditional fuzzy operations for intersection and union (t-norms and t-conorms) are immediately applicable. Specific 
spatial functionality has been added, some examples include the distance between regions and the (fuzzy) surface area of a region [10]. Topology has also been considered [11]; unlike in the crisp case appropriate definitions for the boundary, interior and exterior had to be derived from the initial given fuzzy set. The research is also still ongoing, as we try to find more optimal definitions. This model serves as a theoretical basis, in [7] and [9] we presented models suitable for implementation.

Fig. 1 The concept of a fuzzy region $\tilde{A}$; a fuzzy set over a two dimensional domain. All points belong to some extent to the region; indicated by means of the membership grade. The lower half of the figure shows a cross section. The shades of grey relate to the membership grades: darker shades match higher membership grades (the region has a dark outline to indicate its maximal outline).

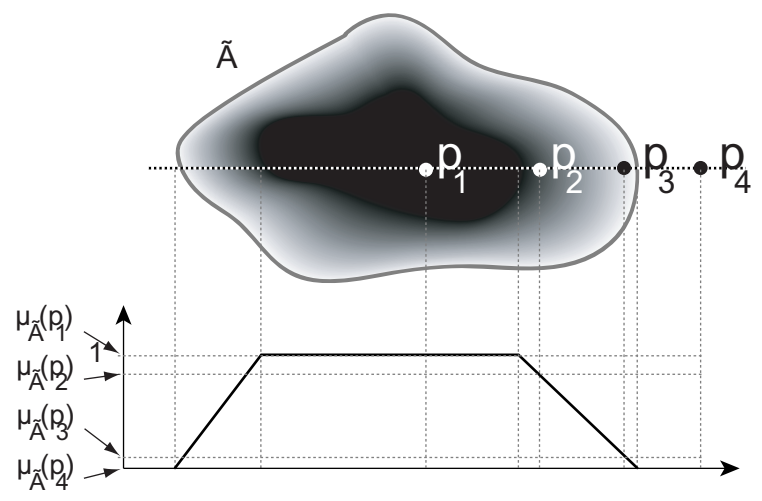

The representation model can be used with a veristic interpretation to yield a fuzzy region: in this interpretation, the membership grades represent the extent to which the points belong to the region; but all points belong to the region. Giving the membership grades a possibilistic interpretation results in the representation of a fuzzy point: we are modelling a crisp point, and every element of fuzzy region is a candidate with its membership grade indicating the possibility. It goes without saying that the operators (e.g. distance) are impacted by this. In either interpretation, all points are considered to be independent of one another. In some situations, a user has added knowledge about the fact some points are linked (e.g. the example of the lake). A first extension to overcome this, makes use of the concept of the powerset.

\subsubsection{Powerset extension}

To overcome the problem that all points are considered independently, elements of the fuzzy region need to be grouped. For this we first look at the concept: the fuzzy region is a fuzzy set of $\mathbb{R}^{2}$. To define the extension, we need to redefine the domain, and for this the powerset of $\mathbb{R}^{2}$ is used. The powerset $\wp$ of a set is a new set containing all the possible subsets of that particular set. To illustrate this, consider the following example:

$$
\wp(\{0,1,2\})=\{\{\},\{0\},\{1\},\{2\},\{0,1\},\{0,2\},\{1,2\},\{0,1,2\}\}
$$

For $\mathbb{R}^{2}$ this becomes: 


$$
\wp\left(\mathbb{R}^{2}\right)=\left\{X \mid X \subseteq \mathbb{R}^{2}\right\}
$$

Definition 2 shows the definition for fuzzy regions using the above powerset, as defined in [12].

Definition 2 (Fuzzy region with powerset extension).

$$
\tilde{R}=\left\{\left(P, \mu_{\tilde{R}}(P)\right) \mid P \in \wp\left(\mathbb{R}^{2}\right) \wedge \forall P_{1}, P_{2} \in \tilde{R}: P_{1} \cap P_{2}=\emptyset\right\}
$$

Note that the intersection between any two elements should be empty: it is required that no two elements of the fuzzy region share points. A point can only be considered to belong to the region once, even if it is to a membership grade less than 1 . When a fuzzy region is defined by means of a limited number of subregions, the concept bears resemblance to the concept of plateau regions [3]. The operations distance and surface have been considered in [12]. The extended concept of fuzzy regions is illustrated on figure 2, we refer to [12] for more details on the surface area. Simply put, the fuzzy surface area represents every possible surface area; this implies that when points are grouped in a subregion (e.g. the regions $\tilde{B}$ and $\tilde{C}$, there are less possibilities: the subregions either count as a whole, or do not count at all.

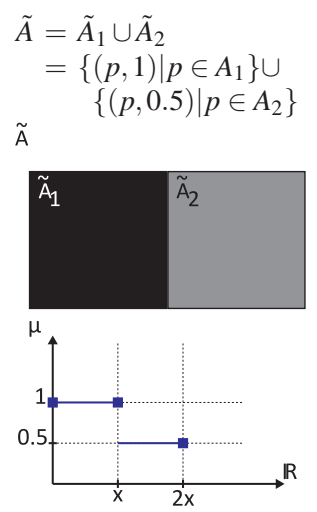

(a)
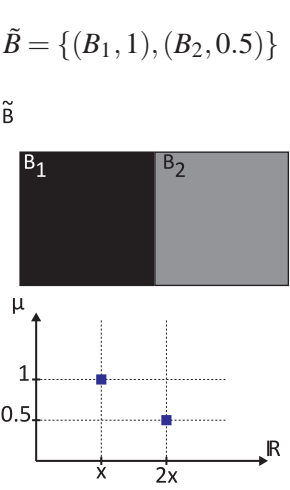

(b)
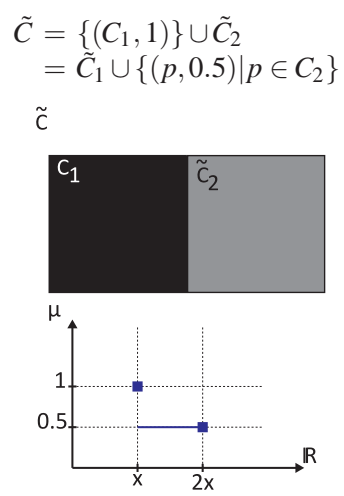

(c)

Fig. 2 Three different examples of fuzzy regions, with their surface areas: (a) the classical model, where each points is treated independently, (b) the extension with the powerset, showing a region consisting of two subregions each counted as a whole, (c) a region where there is both a subregion where points are treated independently, and a subregion that is counted as a whole. For each region, a mathematical explanation of its elements, a graphical illustration, where the shade of grey is representative of the membership grade, and its fuzzy surface area are shown.

\subsubsection{Example}

The fuzzy region based on the powerset still has the same interpretations as before. The lake from the example can now be represented as a fuzzy region, but with all 
the points that are at the same altitude (and thus would flood at the same time) contained within a subregion. While at first sight this makes no real difference to the representation of the region, this change in definition impacts its use. The fuzzy surface area [12] of the lake for instance will now be represented more accurately, possibly having less possible values than before, as points at the same altitude are not counted individually but as a group.

\subsection{Limitations}

While powerset extension mentioned in 2.1.2 allows for a richer model, it still is not without its limitations.

The first limitation is that all points contained within a subregion are required to have the same membership grade. This is basically caused by the fact that the subregion is a crisp region, which is given a membership grade as a whole. Additional knowledge could be present so that it may be necessary to group points with different membership grades.

The second limitation is more subtle and concerns the interpretations. Consider the example of the lake: it is said that the membership grades carry a veristic interpretation; all the points or subregions in the case of the powerset extension belong to the region to some extent. However, when we consider the changing water level on the lake, we could consider it to have a possibilistic interpretation, as there is only one water level at a time (it is just unknown to us for some reason). On the other hand, it is not possible to give the fuzzy region the possibilistic interpretation, as this would not represent a region anymore but more a fuzzy point (and a fuzzy set of candidate locations).

To overcome both limitations, an improved version of the powerset extension is presented.

\section{Fuzzy powerset extension}

\subsection{Concept}

The limitations of the previous extension were mentioned earlier. The first limitation, lack of grouping together points with different membership grades, could be solved by using fuzzy subregions. The use of fuzzy subregions will however introduce a second membership grade for the points of the region, which could serve as a solution to the interpretation problem.

The concept of the fuzzy powerset extension is similar to the previous extension: a fuzzy region will now be defined as a fuzzy set of fuzzy sets, which is achieved using the fuzzy powerset. The fuzzy powerset $\tilde{\wp}$ of a set $A$ is the set of of all fuzzy 
sets over the given set $A$.

$$
\tilde{\S}(A)=\left\{\tilde{X} \mid \forall x: \mu_{\tilde{x}}(x)>0 \Rightarrow x \in A\right\}
$$

By using the $\tilde{\delta}\left(\mathbb{R}^{2}\right)$ as the domain for the fuzzy region, a region with fuzzy subregions can be defined.

\subsection{Definition}

Using the fuzzy powerset, it is possible to define a fuzzy region similarly as has been done with the powerset.

Definition 3 (Level-2 fuzzy region).

$$
\tilde{R}=\left\{\left(\tilde{R}^{\prime}, \mu_{\tilde{R}}\left(\tilde{R}^{\prime}\right)\right) \mid \tilde{R}^{\prime} \in \tilde{\wp}\left(\mathbb{R}^{2}\right)\right\}
$$

The membership function is defined as:

$$
\begin{aligned}
\mu_{\tilde{R}}: \tilde{\S}\left(\mathbb{R}^{2}\right) & \mapsto[0,1] \\
\tilde{R}^{\prime} & \rightarrow \mu_{\tilde{R}}\left(\tilde{R}^{\prime}\right)
\end{aligned}
$$

The elements of the fuzzy region $\tilde{R}$ are fuzzy regions as per definition 1; an important difference with the previous definition is that we now allow different subregions to share elements. The definition comprises what is referred to as a level-2 fuzzy set: a fuzzy set defined over a fuzzy domain ([2],[4]) and is named accordingly. This concept is not to be confused with a type-2 fuzzy set ([5]), which is a fuzzy set defined over a crisp domain but where the membership grades are fuzzy sets.

On figure 3, a region $\tilde{R}$ defined with three fuzzy subregions is shown; $\tilde{R}=$ $\left\{\left(\tilde{R}_{1}^{\prime}, \mu_{\tilde{R}}\left(\tilde{R}_{1}^{\prime}\right)\right),\left(\tilde{R}_{2}^{\prime}, \mu_{\tilde{R}}\left(\tilde{R}_{2}^{\prime}\right)\right),\left(\tilde{R}_{3}^{\prime}, \mu_{\tilde{R}}\left(\tilde{R}_{3}^{\prime}\right)\right)\right\}$.

Fig. 3 A fuzzy region as $\tilde{R}$ defined using definition 3. $\tilde{R}$ has three overlapping subregions $\left(\tilde{R_{1}^{\prime}}, \tilde{R_{2}^{\prime}}\right.$ and $\left.R_{3}^{\prime}\right)$; each fuzzy region (1), as indicated by the grey scales. These subregions are candidate representations for the feature modelled, and carry membership grades to indicate this possibility (not shown). As before, darker colours indicate higher membership grades.

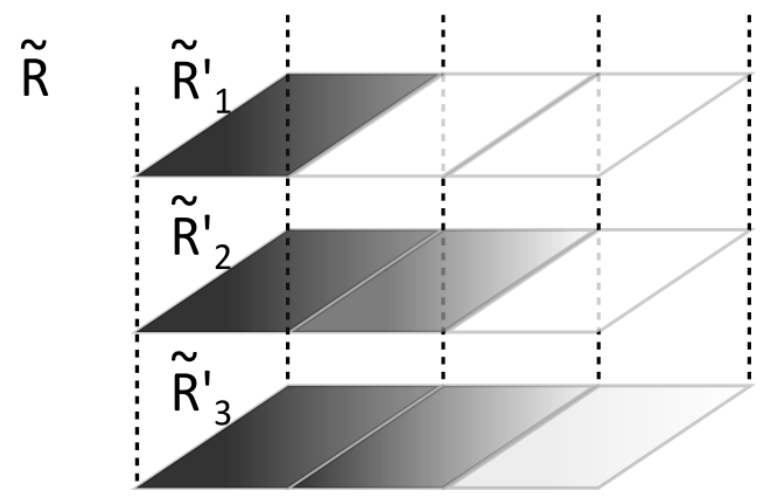




\subsection{Interpretation}

By using fuzzy regions (definition 1) as basic elements for the new concept, points of the universe will have multiple membership grades associated: some from their own membership in the subregion, some from the membership of the subregion that contains them. To explain the interpretations, first the new definition will be considered under the same limitation as the powerset extension: subregions will not be allowed to overlap. This simplification has quite an impact on the interpretation, but makes the link with the previous extension more obvious.

\subsubsection{Non overlapping subregions}

In the first extension (section 2.1.2), subregions were not allowed to overlap in order to simplify the model. In this section, we will consider the new extension (section 3) under the same limitation, merely to illustrate the way it impacts the interpretation. By limiting the definition in not allowing overlapping subregions, it becomes a straight forward extension of the previous definition (2.1.2): the fuzzy set over the new domain (i.e. the region $\tilde{R}$ ) needs to have a veristic interpretation as it is represents a region (all elements belong to it). The fuzzy set in each subregion $\tilde{R}^{\prime}$ also needs to carry a veristic interpretation (or the subregion would not be a correct representation of a region). An example of such a region is shown on figure 4.

Fig. 4 Illustration of a fuzzy region as $\tilde{A}$ defined using the above definition 3 , but not allowing regions to overlap. It shows three subregions $\left(\tilde{A_{1}^{\prime}}, \tilde{A_{2}^{\prime}}\right.$ and $\left.\tilde{A_{3}^{\prime}}\right)$. Each of these subregions is a fuzzy region (using definition 1) on its own, as indicated by the grey scales. The feature being modelled is the union of all these subregions; and therefore contains each of them to some extent. Each subregion carries a membership grade to indicate this extent. As before, darker colours indicate higher membership grades.

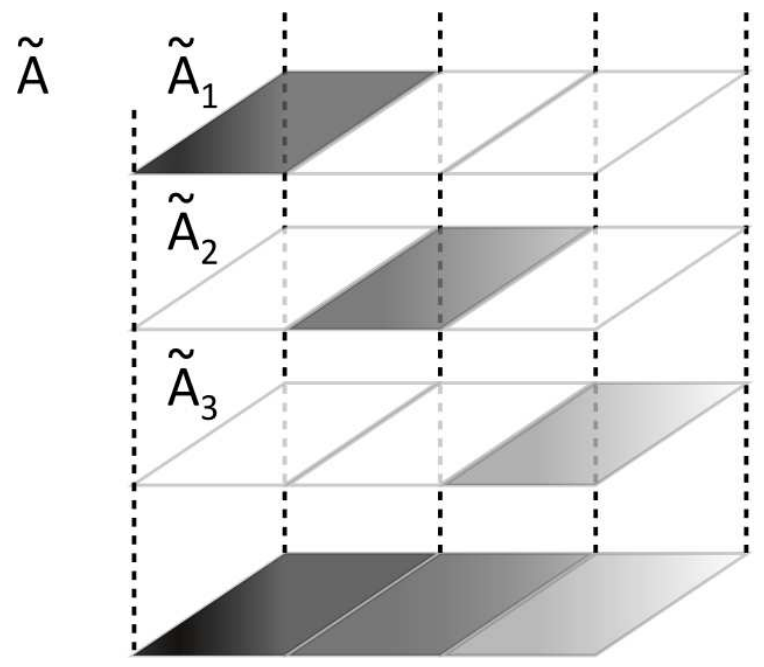

Points $p$ of the universe $\mathbb{R}^{2}$ carry only two membership grades, as they only belong to one $\tilde{R}^{\prime} \in \tilde{R}$ : the membership grade $\mu_{(R)}\left(R^{\prime}\right)$ and indirectly $\mu_{\left(R^{\prime}\right)}(p)$. To say something about $p$ in relation to $\tilde{R}$, these grades can be accumulated, using the intersection of the membership grades of the point in the subregion, and the membership 
grade of the subregion. This allows us to define the function $\mu_{\tilde{R}}^{\prime}$ which returns the membership for individual points of the universe (it is not a true membership function, we will elaborate on this in the next section).

Despite the presence of the limitation, the first problem described in 2.2 is solved: points with different membership grades can be grouped in one subregion. The second problem however, the ambiguity regarding the interpretation, remains.

\section{Example}

To simplify the example, the regions will be defined as finite sets of points. The formulas also hold for infinite sets. Consider the region $\tilde{R}$ defined as below.

$$
\tilde{R}=\left\{\left(\tilde{R}_{1}^{\prime}, 1\right),\left(\tilde{R}_{2}^{\prime}, 0.5\right)\right\} \text { with }\left\{\begin{array}{l}
\tilde{R}_{1}^{\prime}=\left\{\left(p_{1}^{1}, 0.8\right),\left(p_{2}^{1}, 0.6\right)\right\} \\
\tilde{R}_{2}^{\prime}=\left\{\left(p_{1}^{2}, 0.8\right),\left(p_{2}^{2}, 0.4\right)\right\}
\end{array}\right.
$$

Using a t-norm $T$, the membership grades $\mu^{\prime}$ are

$$
\begin{aligned}
& \mu_{\tilde{\tilde{N}}}^{\prime}\left(p_{1}^{1}\right)=T\left(\mu_{\tilde{R}}\left(\tilde{R}_{1}^{\prime}\right), \mu_{\tilde{R}_{1}^{\prime}}\left(p_{1}^{1}\right)\right)=T(1,0.8) \\
& \mu_{\tilde{R}}^{\prime}\left(p_{2}^{1}\right)=T\left(\mu_{\tilde{R}}\left(\tilde{R}_{1}^{\prime}\right), \mu_{\tilde{R}_{1}^{\prime}}\left(p_{2}^{1}\right)\right)=T(1,0.6) \\
& \mu_{\tilde{R}}^{\prime}\left(p_{1}^{2}\right)=T\left(\mu_{\tilde{R}}\left(\tilde{R}_{2}^{\prime}\right), \mu_{\tilde{R_{1}^{\prime}}}\left(p_{1}^{2}\right)\right)=T(0.5,0.8) \\
& \mu_{\tilde{R}}^{\prime}\left(p_{2}^{2}\right)=T\left(\mu_{\tilde{R}}\left(\tilde{R}_{2}^{\prime}\right), \mu_{\tilde{R}_{1}^{\prime}}\left(p_{2}^{2}\right)\right)=T(0.5,0.4)
\end{aligned}
$$

\subsubsection{Overlapping subregions}

For regions with overlapping subregions, the situation becomes more complex. Each of the subregions $\tilde{R}^{\prime}$ can be seen as a candidate fuzzy region, but as a region has a veristic interpretation of its elements. Each subregion $\tilde{R}^{\prime}$ is a basic element of a fuzzy set $\tilde{R}$, in which it will carry a membership grade $\mu_{\tilde{R}}\left(\tilde{R}^{\prime}\right)$ to indicate its possibility, and as such a possibilistic interpretation is needed on this second level. The model can thus be used to represent fuzzy points as well: it suffices to consider singleton sets subregions. We can now consider the relationship between a single point $p$ of the universe $\mathbb{R}^{2}$ with the fuzzy region $\tilde{R}$. While it is of course not possible to consider a point of a subregion $\tilde{R}^{\prime}$ independently from other points of the same subregion $\tilde{R}^{\prime}$, it is possible to provide information regarding individual points. A point of the universe, if it is contained within more than one subregion, will have a number of membership grades from the first level and a number of membership grades from the second level. The former relate to the extent to which the point belongs to the region, whereas the latter relate to the possibility the point belongs to the region. Expressing the membership of this point in relation to the fuzzy region $\tilde{R}$ implies there is uncertainty about the points membership grade. To express this, we can resort to a fuzzy set to describe the membership. 


\section{Definition 4 (Membership of points).}

$$
\begin{aligned}
\mu_{\tilde{R}}^{\prime}(p): \mathbb{R}^{2} & \mapsto \widetilde{[0,1]} \\
p & \rightarrow \mu_{\tilde{R}}(p)
\end{aligned}
$$

The membership function is defined as

$$
\mu_{\tilde{R}}^{\prime}(p)=\bigcup_{\tilde{R}^{\prime} \in \tilde{R}}\left\{\left(\mu_{\tilde{R}^{\prime}}^{\prime}(p), \mu_{\tilde{R}}\left(\tilde{R}^{\prime}\right)\right)\right\}
$$

Note that $\mu_{\tilde{R}}^{\prime}(p)$ is not a true membership function, as $\tilde{R}$ is not a set of points. The function is also not normalized: a point can belong to an extent smaller than 1 to regions that have a possibility smaller than 1 . From this definition, it can be seen that while the region was defined as level-2 fuzzy set (fuzzy set over $\tilde{\delta}\left(\mathbb{R}^{2}\right)$ ); we can consider it a type- 2 fuzzy set (over $\mathbb{R}^{2}$ ), but in this latter view we loose the knowledge about the subregions. The value of this function is that it allows us to make statements on individual points of the universe in relation to a fuzzy region.

\section{Example}

To represent the region, all the outlines for the possible boundary need to be considered, along with how possible each outline is (some outlines may be quite likely, whereas others are not). The outline can have points with different membership grades to indicate an imprecise outline. A simple example using finite sets to illustrate the functions is given below.

$$
\tilde{R}=\left\{\left(\tilde{R}_{1}^{\prime}, 1\right),\left(\tilde{R}_{2}^{\prime}, 0.6\right),\left(\tilde{R}_{3}^{\prime}, 0.4\right)\right\} \text { with }\left\{\begin{array}{l}
\tilde{R}_{1}^{\prime}=\left\{\left(p_{1}, 1\right),\left(p_{2}, 0.7\right)\right\} \\
\tilde{R}_{2}^{\prime}=\left\{\left(p_{1}, 0.8\right),\left(p_{2}, 0.7\right)\right\} \\
\tilde{R}_{3}^{\prime}=\left\{\left(p_{1}, 0.4\right),\left(p_{3}, 0.8\right)\right\}
\end{array}\right.
$$

The membership grades for the three points then are:

$$
\begin{aligned}
\mu_{\tilde{R}}^{\prime}\left(p_{1}\right) & =\left\{\left(\mu_{\tilde{R}_{1}^{\prime}}^{\prime}\left(p_{1}\right), \mu_{\tilde{R}}^{\prime}\left(\tilde{R}_{1}^{\prime}\right)\right)\right\} \cup\left\{\left(\mu_{\tilde{R}_{2}^{\prime}}^{\prime}\left(p_{1}\right), \mu_{\tilde{R}}^{\prime}\left(\tilde{R}_{2}^{\prime}\right)\right\} \cup\left\{\left(\mu_{\tilde{R}_{3}^{\prime}}^{\prime}\left(p_{1}\right), \mu_{\tilde{R}}^{\prime}\left(\tilde{R}_{3}^{\prime}\right)\right)\right\}\right. \\
& =\{(1,1),(0.8,0.6),(0.4,0.4)\} \\
\mu_{\tilde{R}}^{\prime}\left(p_{2}\right) & =\left\{\left(\mu_{\tilde{R}_{1}^{\prime}}^{\prime}\left(p_{2}\right), \mu_{\tilde{R}}^{\prime}\left(\tilde{R}_{1}^{\prime}\right)\right)\right\} \cup\left\{\left(\mu_{\tilde{R}_{2}^{\prime}}^{\prime}\left(p_{2}\right), \mu_{\tilde{R}}^{\prime}\left(\tilde{R}_{2}^{\prime}\right)\right)\right\}=\{(0.7, S(1,0.6))\}=\{(0.7,1)\} \\
\mu_{\tilde{R}}^{\prime}\left(p_{3}\right) & =\left\{\left(\mu_{\tilde{R}_{3}^{\prime}}^{\prime}\left(p_{3}\right), \mu_{\tilde{R}}^{\prime}\left(\tilde{R}_{3}^{\prime}\right)\right)\right\}=\{(0.8,0.4)\}
\end{aligned}
$$

Each fuzzy membership has a possibilistic interpretation to indicate the possibility the element belongs to the region to the given extent. The point $p_{1}$ belongs to an extent 1 with a possibility of 1 (if it is in subregion $\tilde{R}_{1}^{\prime}$ ), to an extent 0.8 with possibility 0.6 (subregion ${\tilde{R_{2}^{\prime}}}_{\text {) }}$ and to an extent 0.4 with possibility 0.4 (subregion $\tilde{R}_{3}^{\prime}$ ). For $p_{2}$ an s-norm is needed as it belongs to the same extent to two regions; in this example the maximum was used. 


\section{Conclusion}

In this contribution, we presented an important extension to our model for fuzzy regions, yielding level-2 fuzzy regions. The extension allows for two levels of uncertainty or imprecision, allowing the representation of features that partly belong to the region, and features that possibly belong to the region (or any combination). This change makes for a much richer modelling, allowing the fuzzy regions to represent real life features more closely. The extension also unifies the representation of fuzzy regions and fuzzy points, overcoming the need of specifying the interpretation. Obviously the changes require adaptation of a number of operations that have been defined so far, which is our main focus of the future work.

\section{References}

1. Dubois D., Prade H.: Fundamentals of Fuzzy Sets. Kluwer Academic Publishers (2000)

2. Gottwald S.: Set theory for fuzzy sets of higher level. Fuzzy Sets and Systems, Volume 2, Issue 2, pp. 125-151 (1979)

3. Kanjilal V., Liu H., Schneider M.: Plateau regions: An implementation concept for fuzzy regions in spatial databases and GIS. In Hüllermeier E; Kruse, R; Hoffmann, F (Eds.), in Lecture Notes in Artificial Intelligence 6178, pp. 624-633, (2010).

4. Klir G. J., Yuan B.: Fuzzy sets and fuzzy logic: Theory and applications. New Jersey: Prentice Hall, (1995)

5. Mendel J. M.: Uncertain Rule-Based Fuzzy Logic Systems, Introduction and New Directions; Prenctice Hall PTR, (2001).

6. Rigaux P., Scholl M., Voisard A.: Spatial Databases with Applications to GIS. Morgan Kaufman Publishers (2002)

7. Verstraete J., De Tré G., Hallez A.: Adapting TIN-layers to Represent Fuzzy Geographic Information. The 7th Meeting of the EURO Working Group on Fuzzy Sets, pp. 57-62 (2002)

8. Verstraete J., De Tré G., De Caluwe R., Hallez A.: Field Based Methods for the modelling of Fuzzy Spatial Data. Fuzzy modeling with Spatial Information for Geographic Problems, eds. Petry F., Robinson v., Cobb M., 41-69, Springer-Verlag, (2005)

9. Verstraete J., Hallez A., De Tré G.: Bitmap Based Structures for the modelling of Fuzzy Entities. Special issue of Control \& Cybernetics vol. 35 no. 1, 147-164, (2006)

10. Verstraete J.: Fuzzy regions: interpretations of surface area and distance. Control and Cybernetics, 38, 509-528, (2009).

11. Verstraete J. A quantitative approach to topology for fuzzy regions. Rutkowski, L.; Scherer, R.; Tadeusiewicz, R.; Zadeh, L.A.; Zurada, J.M. (Eds.). Artificial Intelligence and Soft Computing, Part I, Lecture Notes in Artificial Intelligence vol. 6113. (10th ICAISC, Zakopane, Poland, June 13-17), pp. 248-255, (2010).

12. Verstraete J.: Fuzzy regions: adding subregions and the impact on surface and distance calculation. In Hüllermeier E; Kruse, R; Hoffmann, F (Eds.), Communications in Computer and Information Science, Vol. 80, Part 1., pp. 561-570, (2010).

13. Zadeh L.A.: Fuzzy Sets. Information and Control 1 3, 338-353 (1965)

14. Zimmerman H-J.: Practical Applications of Fuzzy Technologies. Kluwer Academic Publishers (1999) 